\title{
A Framework for Modeling and Optimizing Maintenance in Systems Considering Epistemic Uncertainty and Degradation Dependency based on PDMPs
}

\begin{abstract}
A modeling and optimization framework for the maintenance of systems under epistemic uncertainty is presented in this paper. The component degradation processes, the condition-based preventive maintenance and the corrective maintenance are described through piecewise-deterministic Markov processes in consideration of degradation dependency among degradation processes. Epistemic uncertainty associated with component degradation processes, is treated by considering interval-valued parameters. This leads to the formulation of a multi-objective optimization problem whose objectives are the lower and upper bounds of the expected maintenance cost, and whose decision variables are the periods of inspections and the thresholds for preventive maintenance. A solution method to derive the optimal maintenance policy is proposed by combining finite-volume scheme for calculation, differential evolution and non-dominated sorting differential evolution for optimization. An industrial case study is presented to illustrate the proposed methodology.
\end{abstract}

Index Terms-Maintenance optimization, epistemic uncertainty, degradation dependency, multi-objective optimization, piecewise-deterministic Markov process.

\section{NOTATIONS}

$L_{m} \quad$ Degradation process characterized by a PBM

$K_{n} \quad$ Degradation process characterized by a MSM

$\boldsymbol{D}_{O_{q}} \quad$ Degradation sate of component $O_{q}$

$\boldsymbol{X}_{L_{m}}(t) \quad$ State variable of $L_{m}$

$\boldsymbol{X}_{L_{m}}^{\boldsymbol{D}}(t) \quad$ Degradation variables of $L_{m}$

$\boldsymbol{X}_{L_{m}}^{\boldsymbol{P}}(t) \quad$ Physical variables of $L_{m}$

$\mathcal{F}_{L_{m}} \quad$ Failure states set of $L_{m}$

$Y_{K_{n}}(t) \quad$ State variable of $K_{n}$

$\boldsymbol{S}_{K_{n}} \quad$ State set of $K_{n}$

$\mathcal{F}_{K_{n}} \quad$ Failure states set of $K_{n}$

$\boldsymbol{\theta}_{\boldsymbol{K}} \quad$ Parameters in MSMs of the system

$\lambda_{i}\left(j \mid \boldsymbol{X}(t), \boldsymbol{\theta}_{\boldsymbol{K}}\right) \quad$ Transition rate from state $i$ to $j$

$\boldsymbol{\theta}_{\boldsymbol{L}} \quad$ Parameters in PBMs of the system

$\boldsymbol{f}_{\boldsymbol{L}}\left(\boldsymbol{Z}_{k}(t), t \mid \boldsymbol{\theta}_{\boldsymbol{L}}\right) \quad$ Deterministic physics equations in $\boldsymbol{L}$

$\boldsymbol{Z}(t) \quad$ Degradation state of the system

$\mathcal{F} \quad$ System failure state set

$\boldsymbol{H}_{i} \quad$ Predefined state set of PM for degradation process $i$

$I_{A} \quad$ Inspection task for degradation processes constituting $\boldsymbol{A}$

$T_{A} \quad$ Fixed period of inspection task $I_{A}$

$N_{m} \quad$ Number of maintenance tasks experienced by the system

$T_{\text {miss }} \quad$ System mission time

$T_{m}^{k} \quad$ Execution time of the $k$-th maintenance task

$\boldsymbol{Z}_{k}(t) \quad$ Degradation state of the system defined on $\left[T_{m}^{k-1}, T_{m}^{k}\right]$

$C(t) \quad$ Maintenance cost

$C_{I_{A}} \quad$ Cost of the inspection task $I_{A}$

$C_{P}^{O_{q}} \quad$ Cost of PM to component $O_{q}$
$N_{P}^{O_{q}}(t, \boldsymbol{H}, \boldsymbol{T} \mid \boldsymbol{\theta}) \quad$ Number of PM tasks to component $O_{q}$ until time $t$

$N_{D}^{O_{q}}\left(t, \boldsymbol{H}, \boldsymbol{T} \mid \boldsymbol{\theta}, \boldsymbol{x}_{\boldsymbol{L}}^{*}\right) \quad$ Number of CM tasks to component $O_{q}$ until time $t$

$C_{F} \quad$ Penalty cost of experiencing a system failure

$N_{F}\left(t, \boldsymbol{H}, \boldsymbol{T} \mid \boldsymbol{\theta}, \boldsymbol{x}_{\mathbf{L}}^{*}\right)$ Number of system failures until time until time $t$

$p_{t}^{\boldsymbol{Z}_{k}}(d \boldsymbol{z} \mid \boldsymbol{\theta}) \quad$ Probability distribution of $\boldsymbol{Z}_{k}(t)$

$\boldsymbol{z}_{O_{q}} \quad$ Degradation state of the component $O_{q}$ in $\boldsymbol{Z}$

$\boldsymbol{H}_{O_{q}} \quad$ State set for PM of the component $O_{q}$

$\boldsymbol{T}^{O_{q}} \quad$ State set for PM of the component $O_{q}$

\section{INTRODUCTION}

$\mathrm{M}$ AINTENANCE contributes to ensuring the safe and efficient operation of industrial systems [1]. The contribution to safety especially is in highly hazardous industries, such as the nuclear and aerospace ones. The interactions among components complicate the modeling for maintenance planning, which becomes a big challenge and have been categorized by Thomas [2] into the following groups: economic, structural and stochastic dependencies. The economic dependence exists when the cost of maintaining several components simultaneously is different from that of maintaining them individually. The structural dependence occurs if some working components need to be removed or replaced to execute the maintenance of the failed ones. The stochastic dependence, applies when the one component state may affect other components states or their failure rates. Failure interactions [3] have been the most discussed cases for stochastic dependence, which indicates that one component failure can influence the failure rates of other components, or lead them to failures with certain probabilities.

In practice, the failure of industrial components is often the result of multiple and possibly dependent mechanisms (e.g. bearings wear due to friction and impeller wear due to cavitation and erosion, can both lead to centrifugal pump failure). Dependency among degradation mechanisms or processes has received less attention within the framework of maintenance modeling and optimization of multi-component systems. Özekici [4] has considered interdependent aging processes between components due to continuous wear and shocks, and proposed an optimal periodic replacement policy. Rasmekomen and Parlikad [3] have considered degradation dependency in terms of output performance between one critical component and other parallel components based on aging processes, and studied the optimal age-based maintenance policy. Hong et al. [5] have used copulas to model degradation dependency among all the components of a system 
and obtained the optimal maintenance policy including condition-based preventive maintenance (PM) with periodic inspections and instantaneous corrective maintenance $(\mathrm{CM})$. Van Horenbeek and Pintelon [6] have proposed a dynamic predictive maintenance policy that minimizes the long-term mean maintenance cost per unit time in consideration of economic, structural and stochastic dependence.

To describe component degradation processes, a number of models have been developed. They can be mainly classified into the following groups: (1) statistical distributions, based on failure/life time data (e.g. Weibull distribution [11], Bernstein distribution [12]); (2) stochastic processes, describing the evolution of one or more degradation parameters by gradual degradation increments over time, and the failure occurs when the degradation parameter values reach predefined thresholds (e.g. Gamma process [13], Wiener process [14], inverse Gaussian process [15]); (3) multi-state models (MSMs), describing by finite degradation states of the underlying degradation process (e.g. Markov model [16]) and (4) physics-based models (PBMs), based on the material science or structure knowledge of the physics of degradation (e.g. mass and energy balances based models [17]). In this work, we focus on critical components with high reliabilities such as the nuclear and aerospace ones. Therefore, we mainly consider PBMs and MSMs, since degradation/failure data of highly reliable components are always insufficient to estimate statistical distributions or stochastic processes. Recently, a modeling approach employing a piecewise-deterministic Markov process (PDMP) has been proposed and developed in [18] to integrate PBMs and MSMs for dealing with the degradation dependencies among degradation processes of highly reliable components.

An issue that arises in degradation modeling is epistemic uncertainty, because of the imprecise or incomplete information of component degradation processes, especially for the highly reliable ones. The values of the parameters of the physics equations (e.g. wear coefficients), influencing factors (e.g. temperatures and pressures) or transition rates between different degradation states may be poorly known and inferred from the scarce data available and from elicited expert judgment [15]. This uncertainty must be reflected in the modeling and accounted for in the maintenance optimization that rests on it. Fuzzy sets have been employed to mathematically represent epistemic uncertainty in some works $[16,17]$. However, determining appropriate membership functions may be a difficult task in practice. In these cases, intervals can be used as a more general and less knowledge and information demanding representation of uncertainty than fuzzy sets [18].

In practice, the failures of industrial components are often the results of multiple and possibly competing mechanisms (e.g. friction-induced wear of the bearings and impeller wear caused by cavitation and erosion by the flow, can both lead to centrifugal pump failure). For multi-component systems, the dependencies among these mechanisms within one component (e.g. the wear of rubbing surfaces influenced by the environmental stress shock within a micro-engine), or/and among different components (e.g. the degradation of the pre-filtrations stations leading to a lower performance level of the sand filter in a water treatment plant) need to be considered.
Additionally, in industrial systems, many critical components (such as pumps and valves in nuclear and aerospace industries) are designed to be highly reliable in order to avoid possible human and economic losses. As a result, their degradation and/or failure data are often limited. Therefore, epistemic uncertainties due to incomplete or imprecise knowledge is unavoidable. The values of the parameters of the physics functions (e.g. wear coefficients), influencing factors (e.g. temperatures and pressures) or transition rates between different degradation states may be unknown and inferred from the scarce data and from elicited expert judgment. This uncertainty must be reflected in the modeling and accounted for in the maintenance optimization that rests on it. However, previous works on maintenance modeling and optimization have not considered simultaneously these two important aspects under a single framework, to the knowledge of the authors. In this paper, the component degradation processes, the condition-based preventive maintenance with periodic inspections and the corrective maintenance are modeled by piecewise-deterministic Markov processes in consideration of degradation processes dependency, then, the optimal maintenance policy for multi-component systems has been derived. In [27], a PDMP modeling approach has been proposed with focus on the quantification of systems reliability under degradation dependency and epistemic uncertainty represented by fuzzy sets. In this work, we focus on the modeling and optimization of maintenance for the systems under degradation dependency and epistemic uncertainty. The epistemic uncertainty is represented by interval values, since they are a more general and less knowledge and information demanding representation of uncertainty than fuzzy sets [18].

The main contributions of this work are two-folds: for maintenance modeling: the modeling approach proposed in [18] is extended by (1) including condition-based PM with periodic inspections and CM, and (2) representing model parameters with epistemic uncertainty by interval values; for maintenance optimization: (1) both the period for inspection tasks and the thresholds for PM are considered as the decision variables in the optimization problem formulation; (2) a new optimization method integrating non-dominated sorting differential evolution (NSDE) [24], differential evolution (DE) [25] and finite-volume (FV) scheme [26] is proposed to derive the optimal maintenance policy by setting the interval-valued expected maintenance cost as the objective function.

The rest of the paper is structured as follows. Section 2 presents the problem investigated in this study. Section 3 presents the original degradation model of the whole system based on PDMP modeling framework without maintenance actions. Section 4 inserts maintenance actions into the degradation model. Section 5 presents the formulation of the maintenance optimization problem under epistemic uncertainty. Section 6 introduces the proposed solution approach for deriving the optimal maintenance policy. Section 7 presents an industrial case study to illustrate the proposed methodology. Section 8 presents the numerical results and analysis. Section 9 presents the conclusions and remarks. 


\section{PROBLEM DESCRIPTION}

We consider a multi-component system made of $Q$ highly reliable components denoted by $O=\left\{O_{1}, O_{2}, \ldots, O_{Q}\right\}$. Multiple degradation processes may be involved in each component, which are possibly dependent. Since there is insufficient degradation/failure data available to build statistical or stochastic models of degradation, PBMs and MSMs are employed. All degradation processes in the system can be classified into two groups: (1) $\boldsymbol{L}=\left\{L_{1}, L_{2}, \ldots, L_{M}\right\}$ modeled by $M$ PBMs; (2) $\boldsymbol{K}=\left\{K_{1}, K_{2}, \ldots, K_{N}\right\}$ modeled by $N$ MSMs, where $L_{m}, m=1,2, \ldots, M$ and $K_{n}, n=1,2, \ldots, N$ are the indices of the degradation processes. The degradation state of a component $O_{q} \in \boldsymbol{O}, q=1,2, \ldots, Q$, is determined by its degradation processes $\boldsymbol{D}_{O_{q}} \subseteq \boldsymbol{L} \cup \boldsymbol{K}$ and the component fails when one of its degradation processes becomes failure. A maintenance policy containing both $\mathrm{CM}$ and PM is considered.

\section{MODEL DESCRIPTION WITHOUT MAINTENANCE}

\section{A. Degradation models}

In this section, PBMs, MSMs and PDMP modeling framework for systems considering degradation dependencies will be introduced, which are the basis of the problem and have been proposed in [27].

\section{1) $P B M s$}

The descriptions of the parameters in PBMs are given as follows [27]:

A degradation process $\boldsymbol{X}_{L_{m}}(t), L_{m} \in \boldsymbol{L}$ in the first group, has $d_{L_{m}}$ time-dependent continuous variables $\boldsymbol{X}_{L_{m}}(t)=$ $\left(x_{L_{m}}^{1}(t), x_{L_{m}}^{2}(t), \ldots, x_{L_{m}}^{d_{L_{m}}}(t)\right) \in \mathbb{R}^{d_{L_{m}}}$. Physics equations $\boldsymbol{X}_{L_{m}}^{\cdot}(t)=\boldsymbol{f}_{L_{m}}\left(\boldsymbol{X}_{L_{m}}(t), t \mid \boldsymbol{\theta}_{L_{m}}\right)$, are used to characterize its evolution, which is a system of first-order differential equations. $\boldsymbol{\theta}_{L_{m}}$ are the environmental and operational factors which can influence the evolution of $\boldsymbol{X}_{L_{m}}(t)=\left(\boldsymbol{X}_{L_{m}}^{\boldsymbol{D}}(t), \boldsymbol{X}_{L_{m}}^{\boldsymbol{P}}(t)\right)$ and they are independent of $\boldsymbol{X}_{L_{m}}(t) . \boldsymbol{X}_{L_{m}}^{\boldsymbol{P}}(t)$ are the physical variables influencing $\boldsymbol{X}_{L_{m}}^{\boldsymbol{D}}(t)$, which may depend on $\boldsymbol{X}_{L_{m}}(t)$.

$\boldsymbol{X}_{L_{m}}(t)$ can be divided into two groups of varaibles $\boldsymbol{X}_{L_{m}}(t)=\left(\boldsymbol{X}_{L_{m}}^{\boldsymbol{D}}(t), \boldsymbol{X}_{L_{m}}^{\boldsymbol{P}}(t)\right):$ (1) $\quad \boldsymbol{X}_{L_{m}}^{\boldsymbol{D}}(t)$ are the non-decreasing degradation variables describing the degradation process (e.g. leak area of the piston of the valve); (2) $\boldsymbol{X}_{L_{m}}^{\boldsymbol{P}}(t)$ are the physical variables influencing $\boldsymbol{X}_{L_{m}}^{\boldsymbol{D}}(t)$ (e.g. speed and strength). As long as one $x_{L_{m}}^{i}(t) \in \boldsymbol{X}_{L_{m}}^{\boldsymbol{D}}(t)$ reaches or exceeds its failure threshold $x_{L_{m}}{ }^{*}$, the generic degradation process $L_{m}$ fails. Let $\mathcal{F}_{L_{m}}$ denote the failure state set of $L_{m}$ and $\boldsymbol{x}_{L_{m}}^{*}$ denote the set of all the failure thresholds of $\boldsymbol{X}_{L_{m}}^{\boldsymbol{D}}(t)$.

2) $M S M s$

The descriptions of the parameters in MSMs are given as follows [27]:

A degradation process, $Y_{K_{n}}(t), K_{n} \in \boldsymbol{K}$ in the second group, its state set is denoted by $S_{K_{n}}=\left\{{ }^{\prime} 0^{\prime},{ }^{\prime} 1^{\prime}, \ldots,{ }^{\prime} d_{K_{n}}{ }^{\prime}\right\}$, varying from perfect functioning state ${ }^{\prime} d_{K_{n}}{ }^{\prime}$ to complete failure state ' 0 '. Transition rate $\lambda_{i}\left(j \mid \boldsymbol{\theta}_{K_{n}}\right)=\lim _{\Delta t \rightarrow 0} P\left(Y_{K_{n}}(t+\Delta t)=j \mid Y_{K_{n}}=\right.$ $\left.i, \boldsymbol{\theta}_{K_{n}}\right) / \Delta t, \forall i, j \in \boldsymbol{S}_{K_{n}}, i>j$, characterize the probability per time unit that the system makes a transition from state $i$ to state $j . \boldsymbol{\theta}_{K_{n}}$ represents environmental factors to $K_{n}$ and the related parameters used in $\lambda_{i}$. Let $\mathcal{F}_{K_{n}}=\{0\}$ denote the failure state set of $K_{n}$.

\section{3) Degradation model of the system}

For system reliability analysis, fault tree analysis (FTA) is always used to identify the combinations of basic events (components failures) leading to system failure, and compute its probability by using minimal cut sets found from the fault tree structure. However, this analysis is conducted under the assumption that all basic events are statistically independent. When basic events can be dependent (due to degradation dependency), the method proposed in [41] by using FTA and binary decision diagrams can be applied to identify the groups of components whose failures are dependent and thus divide the degradation processes of the whole system into several smaller groups which are independent from each other. Inside each group, the dependent degradation processes have to be modeled as a whole for reliability evaluation. To perform this evaluation, PDMP modeling framework integrating PBMs and MSMs for degradation dependency has been proposed in [27].

There are two groups of degradation processes ( $\boldsymbol{L}$ and $\boldsymbol{K}$, respectively modeled by $\mathrm{PBMs}$ and MSMs), degradation dependency may exist within each group and between the two groups.

$$
\text { Let } \boldsymbol{X}(t)=\left(\begin{array}{c}
\boldsymbol{X}_{L_{1}}(t) \\
\vdots \\
\boldsymbol{X}_{L_{M}}(t)
\end{array}\right) \text { and } \boldsymbol{Y}(t)=\left(\begin{array}{c}
Y_{K_{1}}(t) \\
\vdots \\
Y_{K_{N}}(t)
\end{array}\right) \text { represent the }
$$

degradation processes of $\boldsymbol{L}$ and $\boldsymbol{K}$, respectively. $\boldsymbol{X}(t)$ denotes the degradation processes modeled by PBMs. Their evolution is characterized by physics functions in the form of first-order differential equation system, partially parameterized by $\boldsymbol{Y}(t)$ whose holding time and arrival states after transition are both random. Therefore $\boldsymbol{X}(t)$ involves randomness. Nevertheless, the evolution of $\boldsymbol{X}(t)$ between two successive transitions of $\boldsymbol{Y}(t)$ is deterministic, determined by the physics functions. The overall degradation process of the system is presented as

$$
\boldsymbol{Z}(t)=\left(\begin{array}{l}
\boldsymbol{X}(t) \\
\boldsymbol{Y}(t)
\end{array}\right)
$$

PDMPs, then, can be employed to model the degradation dependency in the system. Due to the different nature of PBMs and MSMs, the impacts of degradation dependency on the two groups of degradation processes are characterized as follows: the states of $\boldsymbol{Y}(t)$ may influence the evolution of $\boldsymbol{X}(t)$, the values of $\boldsymbol{X}(t)$ may influence the transition times and transition directions of $\boldsymbol{Y}(t)$ (the detailed formulations are shown in eqs. (2) and (3)). Therefore, the evolution of $\boldsymbol{Z}(t)$ has two parts: (1) the stochastic evolution of $\boldsymbol{Y}(t)$ and (2) the deterministic evolution of $\boldsymbol{X}(t)$ between two successive transitions of $\boldsymbol{Y}(t)$. The former is governed by the transition rates of $\boldsymbol{Y}(t)$, which depends on the values of $\boldsymbol{X}(t)$ and $\boldsymbol{Y}(t)$, as follows:

$$
\begin{gathered}
\lim _{\Delta t \rightarrow 0} P\left(\boldsymbol{Y}(t+\Delta t)=j \mid \boldsymbol{X}(t), \boldsymbol{Y}(t)=i, \boldsymbol{\theta}_{\boldsymbol{K}}=\bigcup_{n=1}^{N} \boldsymbol{\theta}_{K_{n}}\right) \\
/ \Delta t=\lambda_{i}\left(j \mid \boldsymbol{X}(t), \boldsymbol{\theta}_{\boldsymbol{K}}\right) \\
, \forall t \geq 0, i, j \in \boldsymbol{S}, i \neq j
\end{gathered}
$$

The latter is described by physics functions of degradations, which depends on the values of $\boldsymbol{Y}(t)$ and $\boldsymbol{X}(t)$, as follows: 


$$
\begin{aligned}
& \dot{\boldsymbol{X}}(t)=\left(\begin{array}{c}
\dot{\boldsymbol{X}_{L_{1}}}(t) \\
\vdots \\
\boldsymbol{X}_{L_{M}}^{\cdot}(t)
\end{array}\right)=\left(\begin{array}{c}
\boldsymbol{f}_{L_{1}}{ }^{\boldsymbol{Y}(t)}\left(\boldsymbol{X}(t), t \mid \boldsymbol{\theta}_{L_{1}}\right) \\
\vdots \\
\boldsymbol{f}_{L_{M}}{ }^{\boldsymbol{Y}(t)}\left(\boldsymbol{X}(t), t \mid \boldsymbol{\theta}_{L_{M}}\right)
\end{array}\right) \\
&=\boldsymbol{f}_{\boldsymbol{L}}{ }^{\boldsymbol{Y}(t)}\left(\boldsymbol{X}(t), t \mid \boldsymbol{\theta}_{\boldsymbol{L}}=\bigcup_{m=1}^{M} \boldsymbol{\theta}_{L_{m}}\right)
\end{aligned}
$$

Let $\mathcal{F}$ denote the system failure state set. It can be defined according to the structure of the system. Then, the system reliability at mission time $T_{\text {miss }}$ can be obtained as follows:

$$
R\left(T_{\text {miss }}\right)=P\left[\boldsymbol{Z}(s) \notin \mathcal{F}, \forall s \leq T_{\text {miss }}\right]
$$

The system failure state set is dependent on system topology and failure logic. To determine this set, reliability analysis tools such as fault tree can be used to identify the combination of primary failure events leading to system failure.

\section{MODEL DESCRIPTION WITH MAINTENANCE}

For condition-based maintenance modeling, the following studies are reviewed. Wang [7] developed a general wear prediction model based on stochastic filtering and hidden Markov theory wear to predict the state of the monitored plant; the observations are related to the true underlying state of the system which is unobservable. To better describe the relationships between observations and underlying state of the system, Wang and Christer [8] first used the residual life at the time of monitoring as a measure of the state of the unit; a decision model based on the expected cost per unit time has been developed to guide preventive maintenance actions. To cope with heterogeneous populations caused by unavoidable manufacturing defects, Zhang et al. [9] developed an inspection-replacement policy, for which the preventive-replacement time is dynamically determined depending on the information obtained by the inspection. To take into account imperfect maintenance, Zhang et al. [10] proposed an imperfect maintenance model that is applicable to systems whose sensor information can be modeled by stochastic processes.

In this paper, the previous modeling framework [27] is extended by including condition-based preventive maintenance actions with periodic inspections and corrective maintenance, the following assumptions are made based on actual maintenance activities performed in industrial practice:

The PM involves condition-based maintenance tasks based on the information collected through inspections. The inspection task $I_{\boldsymbol{A}}, \forall \boldsymbol{A} \subseteq \boldsymbol{L} \cup \boldsymbol{K}$, related to one or more degradation processes constituting $\boldsymbol{A}$, is carried out with fixed period and a cost is associated with each inspection.

The following assumption is made: If the state of one degradation process $i \in \boldsymbol{L} \cup \boldsymbol{K}$, reported by condition inspection, enters the predefined state set for PM denoted by $\boldsymbol{H}_{i}$, then the component containing this degradation process is replaced and a PM cost is incurred depending on component type. Otherwise, no maintenance action is executed.

Component failure can be detected immediately and the failed component is restored to its initial state by the CM, and a $\mathrm{CM}$ cost is incurred depending on the component type.

The duration of inspection tasks is negligible and all maintenance actions are done instantaneously, compared with the lifetime of the components.
The degradation processes and the maintenance policy of an example system are illustrated in Fig 1, considering a mission time $T_{\text {miss }}$. The system is composed of two components $O_{1}$ and $O_{2} . \boldsymbol{D}_{O_{1}}=\left\{L_{1}\right\}$ and $\boldsymbol{D}_{O_{2}}=\left\{K_{1}\right\} . T_{L_{1}}$ and $T_{K_{1}}$ are the periods of the inspection tasks for $I_{L_{1}}$ and $I_{K_{1}}$, respectively. For $L_{1}, \mathrm{PM}$ is carried out whenever its degradation variable $\boldsymbol{X}_{L_{1}}^{\boldsymbol{D}}(t)$ reaches or exceeds its PM threshold $x_{L_{1}}{ }^{p}$ at the time of inspection. The physical variable $\boldsymbol{X}_{L_{1}}^{\boldsymbol{P}}(t)$ is also initialized immediately after PM is performed. For $K_{1}$, PM is carried out when it is in state ' 1 ' at the time of inspection.

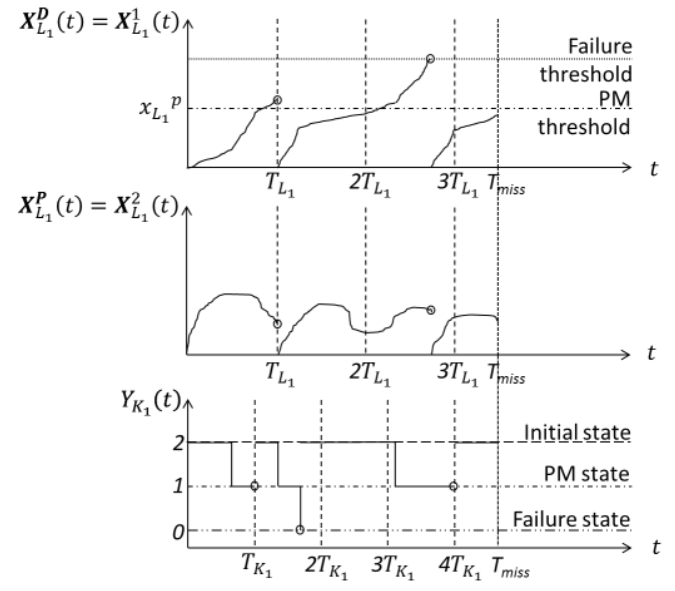

Fig. 1. An illustration of the degradation processes with maintenance policy, for an example system.

To extend the PDMP to model the degradation processes and the maintenance policy, the entire mission time is divided into multiple intervals. In each interval, one new PDMP, $\boldsymbol{Z}_{k}(t), k=1,2, \ldots, N_{m}+1$, is defined, where $N_{m}$ is the number of maintenance tasks the system has experienced till the mission time. Let $T_{m}^{k}, k=1,2, \ldots, N_{m}$ denote the execution time of the $k$-th maintenance task, then $\boldsymbol{Z}_{1}(t)$ is defined on $\left[0, T_{m}^{1}\right], \boldsymbol{Z}_{N_{m}+1}(t)$ is defined on $\left[T_{m}^{N_{m}}, T_{m i s s}\right]$ and $\boldsymbol{Z}_{k}(t), k=$ $2, \ldots, N_{m}$ is defined on $\left[T_{m}^{k-1}, T_{m}^{k}\right]$, respectively. The initial states $\boldsymbol{Z}_{k}(t), k=2, \ldots, N_{m}+1$ are dependent on the maintenance task carried out at time $T_{m}^{k-1}$ and $\boldsymbol{Z}_{k-1}\left(T_{m}^{k-1}\right)$. Fig 2 shows this extension for the degradation processes in Fig 1.

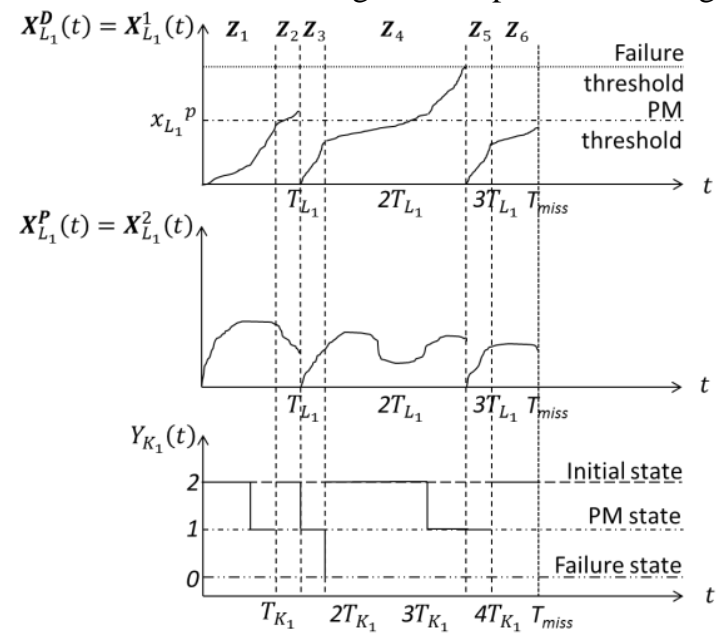

Fig. 2. An illustration of system maintenance, treated via PDMP 
In reality, the two major issues for the maintenance policy are to determine (1) the period $T_{A}, \forall A \subseteq L \cup K$ for each inspection task $I_{A}$ and (2) the state set for PM $\boldsymbol{H}_{i}, \forall i \in \boldsymbol{L} \cup \boldsymbol{K}$ for each degradation process $i$.

\section{MAINTENANCE OPTIMIZATION UNDER UNCERTAINTY}

\section{A. Maintenance optimization criterion}

For the maintenance optimization, the criterion considered is the expected maintenance cost over the system mission time. Let $C(t)$ denote the maintenance cost, $\boldsymbol{H}=\bigcup_{\forall i \in \boldsymbol{L} \cup \boldsymbol{K}} \boldsymbol{H}_{i}$ and $\boldsymbol{T}=\bigcup_{\forall A \subseteq L \cup K} \boldsymbol{T}_{A}, \boldsymbol{\theta}=\boldsymbol{\theta}_{\boldsymbol{L}} \cup \boldsymbol{\theta}_{K}, \boldsymbol{x}_{\boldsymbol{L}}^{*}=\bigcup_{m=1}^{M} \boldsymbol{x}_{L_{m}}^{*}$, for the system functioning until time $t$, we can write:

$$
\begin{gathered}
\mathbb{E}\left(C\left(t, \boldsymbol{H}, \boldsymbol{T} \mid \boldsymbol{\theta}, \boldsymbol{x}_{\boldsymbol{L}}^{*}\right)\right)=\sum_{A \subseteq L \cup K} C_{I_{A}} \cdot\left\lfloor\frac{t}{T_{A}}\right\rfloor+\sum_{o_{q} \in \boldsymbol{O}} C_{P}^{O_{q}} . \\
\mathbb{E}\left(N_{P}^{O_{q}}(t, \boldsymbol{H}, \boldsymbol{T} \mid \boldsymbol{\theta})\right)+\sum_{o_{q} \in \boldsymbol{O}} C_{D}^{O_{q}} \cdot \mathbb{E}\left(N_{D}^{O_{q}}\left(t, \boldsymbol{H}, \boldsymbol{T} \mid \boldsymbol{\theta}, \boldsymbol{x}_{\boldsymbol{L}}^{*}\right)\right)+ \\
C_{F} \cdot \mathbb{E}\left(N_{F}\left(t, \boldsymbol{H}, \boldsymbol{T} \mid \boldsymbol{\theta}, \boldsymbol{x}_{L}^{*}\right)\right)
\end{gathered}
$$

where $C_{I_{A}}$ is the cost of the inspection task $I_{A},\left\lfloor\frac{t}{T_{A}}\right\rfloor$ is the number of times the inspection task $I_{A}$ has been performed until time $t, C_{P}^{O_{q}}$ is the cost of PM performed on component $O_{q}$, $N_{P}^{O_{q}}(t, \boldsymbol{H}, \boldsymbol{T} \mid \boldsymbol{\theta})$ is the number of PM tasks performed on $O_{q}$ until time $t, N_{D}^{O_{q}}\left(t, \boldsymbol{H}, \boldsymbol{T} \mid \boldsymbol{\theta}, \boldsymbol{x}_{\boldsymbol{L}}^{*}\right)$ is the number of CM tasks to $O_{q}$ until time $t, C_{F}$ is the penalty cost of experiencing a system failure and $N_{F}\left(t, \boldsymbol{H}, \boldsymbol{T} \mid \boldsymbol{\theta}, \boldsymbol{x}_{\boldsymbol{L}}^{*}\right)$ is the number of system failures until time $t$

Let $p_{t}^{Z_{k}}(d \boldsymbol{z} \mid \boldsymbol{\theta})$ denote the probability distribution of $\boldsymbol{Z}_{k}(t)$; we, then, can obtain that

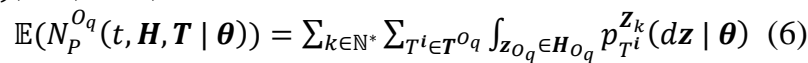
where $\boldsymbol{z}_{O_{q}}$ represents the degradation state of $O_{q}$ in $\boldsymbol{z}, \boldsymbol{H}_{O_{q}}=$ $\cup_{i \in D_{O_{q}}} \boldsymbol{H}_{i}$ denotes the state set for PM of $O_{q}$ and $\boldsymbol{T}^{O_{q}}$ denotes the set of inspection time for $O_{q}$. The function $p_{T^{i}}^{Z_{k}}(d \boldsymbol{z} \mid \boldsymbol{\theta})$ is the probability distribution of $\boldsymbol{Z}_{k}(t)$ at the inspection time $T^{\boldsymbol{i}}$,

$$
\mathbb{E}\left(N_{D}^{O_{q}}\left(t, \boldsymbol{H}, \boldsymbol{T} \mid \boldsymbol{\theta}, \boldsymbol{x}_{\boldsymbol{L}}^{*}\right)\right)=\sum_{k \in \mathbb{N}^{*}} \int_{0}^{t} \int_{\boldsymbol{z}_{O_{q}} \in \mathcal{F}_{O_{q}}} p_{s}^{\boldsymbol{Z}_{k}}(d \boldsymbol{z} \mid \boldsymbol{\theta}) d s \text { (7) }
$$

where $\mathcal{F}_{O_{q}}=\bigcup_{i \in D_{O_{q}}} \mathcal{F}_{i}$ represents the failure state set of $O_{q}$,

$$
\mathbb{E}\left(N_{F}\left(t, \boldsymbol{H}, \boldsymbol{T} \mid \boldsymbol{\theta}, \boldsymbol{x}_{\boldsymbol{L}}^{*}\right)\right)=\sum_{k \in \mathbb{N}^{*}} \int_{0}^{t} \int_{\boldsymbol{z} \in \mathcal{F}} p_{s}^{\boldsymbol{Z}_{k}}(d \boldsymbol{z} \mid \boldsymbol{\theta}) d s
$$

\section{B. Epistemic uncertainty}

Because of the incomplete or imprecise information related to the degradation processes, epistemic uncertainty may exist:

In PBMs: (1) $\boldsymbol{\theta}_{L}$ may be poorly known or elicited through expert judgment; (2) the failure thresholds $\boldsymbol{x}_{\boldsymbol{L}}^{*}$ may be uncertain due to imperfect information.

In MSMs: insufficient degradation and/or failure data may lead to poorly estimated $\boldsymbol{\theta}_{\boldsymbol{K}}$ which determines the transition rates.

The experts in many cases can only confirm an interval of the possible minimum and maximum values of the uncertain transition rate. One practical way of dealing with epistemic uncertainty is to use intervals of values for the uncertain parameters [23]. In this respect, the following assumptions are made (a symbol with an underbar indicates the left limit of that interval, while a symbol with an overbar indicates the right limit of that interval):
The value of $\forall \theta_{i} \in \boldsymbol{\theta}$, is represented by an interval $\left[\theta_{i}\right]=$ $\left[\theta_{i}, \overline{\theta_{i}}\right]$. Let $[\boldsymbol{\theta}]=\bigcup_{\theta_{i} \in \boldsymbol{\theta}}\left[\theta_{i}\right]$.

The value of $\forall x_{L_{m}}^{i}{ }^{*} \in \boldsymbol{x}_{\boldsymbol{L}_{\boldsymbol{m}}}^{*}, \forall L_{m} \in \boldsymbol{L}$, is represented by an interval $\left[x_{L_{m}}^{i}{ }^{*}\right]=\left[\underline{x_{L_{m}}^{i}{ }^{*}}, \overline{x_{L_{m}}^{i}{ }^{*}}\right]$. Let $\left[\boldsymbol{x}_{L_{m}}\right]=U_{x_{L_{m}}^{i}{ }^{*} \in x_{L_{m}}^{*}}\left[x_{L_{m}}^{i}{ }^{*}\right]$ and $\left[\boldsymbol{x}_{\boldsymbol{L}}^{*}\right]=\bigcup_{m=1}^{M}\left[\boldsymbol{x}_{L_{m}}^{*}\right]$.

$\mathbb{E}\left(C\left(t, \boldsymbol{H}, \boldsymbol{T} \mid \boldsymbol{\theta}, \boldsymbol{x}_{\boldsymbol{L}}^{*}\right)\right)$, then, is also an interval, denoted by $\left[\mathbb{E}\left(C\left(t, \boldsymbol{H}, \boldsymbol{T} \mid[\boldsymbol{\theta}],\left[\boldsymbol{x}_{L}^{*}\right]\right)\right)\right]=$

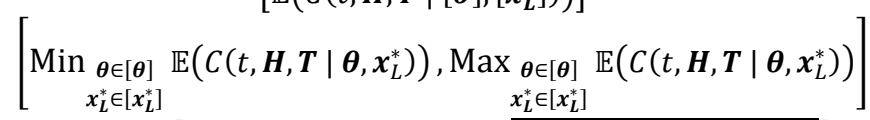

$$
\begin{aligned}
& =\left[\underline{\mathbb{E}\left(C\left(t, \boldsymbol{H}, \boldsymbol{T} \mid[\boldsymbol{\theta}],\left[\boldsymbol{x}_{\boldsymbol{L}}^{*}\right]\right)\right),}, \overline{\mathbb{E}\left(C\left(t, \boldsymbol{H}, \boldsymbol{T} \mid[\boldsymbol{\theta}],\left[\boldsymbol{x}_{\boldsymbol{L}}^{*}\right]\right)\right)}\right](9)
\end{aligned}
$$

\section{Optimization problem}

Based on the models presented above, the problem of maintenance optimization under uncertainty, for mission time $T_{\text {miss }}$, can be defined as:

$$
\begin{gathered}
\operatorname{Min}\left[\mathbb{E}\left(C\left(T_{\text {miss }}, \boldsymbol{H}, \boldsymbol{T} \mid[\boldsymbol{\theta}],\left[\boldsymbol{x}_{\boldsymbol{L}}^{*}\right]\right)\right)\right] \\
\text { Subject to } \boldsymbol{H}_{i} \subseteq W_{i}, \forall i \in \boldsymbol{L} \cup \boldsymbol{K} \\
0 \leq T_{i} \leq T_{\text {miss }}, \forall i \in \boldsymbol{L} \cup \boldsymbol{K}
\end{gathered}
$$

where $W_{i}=\left\{\begin{array}{l}\mathbb{R}^{d_{i}}, \text { if } i \in \boldsymbol{L} \\ \boldsymbol{S}_{i}, \text { if } i \in \boldsymbol{K}\end{array}\right.$

For its solution, it can be reformulated as a multi-objective optimization problem:

$$
\begin{aligned}
& \operatorname{Min} \mathbb{E}\left(C\left(T_{\text {miss }}, \boldsymbol{H}, \boldsymbol{T} \mid[\boldsymbol{\theta}],\left[\boldsymbol{x}_{\boldsymbol{L}}^{*}\right]\right)\right) \\
& \operatorname{Min} \overline{E\left(C\left(T_{\text {miss }}, \boldsymbol{H}, \boldsymbol{T} \mid[\boldsymbol{\theta}],\left[\boldsymbol{x}_{\boldsymbol{L}}^{*}\right]\right)\right)} \\
& \text { Subject to } \boldsymbol{H}_{i} \subseteq W_{i}, \forall i \in \boldsymbol{L} \cup \boldsymbol{K} \\
& 0 \leq T_{i} \leq T_{\text {miss }}, \forall i \in \boldsymbol{L} \cup \boldsymbol{K}
\end{aligned}
$$

where $W_{i}=\left\{\begin{array}{l}\mathbb{R}^{d_{i}}, \text { if } i \in \boldsymbol{L} \\ \boldsymbol{S}_{i}, \text { if } i \in \boldsymbol{K}\end{array}\right.$.

In eq. (11), $\mathbb{E}\left(C\left(T_{\text {miss }}, \boldsymbol{H}, \boldsymbol{T} \mid[\boldsymbol{\theta}],\left[\boldsymbol{x}_{\boldsymbol{L}}^{*}\right]\right)\right)$ indicates the lower bound of the expected maintenance cost whereas $\overline{\mathbb{E}\left(C\left(T_{\text {miss }}, \boldsymbol{H}, \boldsymbol{T} \mid[\boldsymbol{\theta}],\left[\boldsymbol{x}_{\boldsymbol{L}}^{*}\right]\right)\right)}$ indicates the upper bound of the expected maintenance cost. $T_{\text {miss }}$ is the system mission time, $[\boldsymbol{\theta}]$ is the set of uncertain model parameters and $\left[\boldsymbol{x}_{\boldsymbol{L}}^{*}\right]$ is the set of uncertain failure thresholds. The decision variables of the optimization problem are $\boldsymbol{H}$, which is the predefined state set for PM, and $\boldsymbol{T}$, which is the set of periods of the inspection tasks. Eq. (11) means choosing $\boldsymbol{H}_{i}$ (the predefined state set of PM for degradation process $i$ ) and $T_{i}$ (the fixed period of PM for degradation process $i$ ) to minimize the lower and upper bounds of the expected maintenance cost simultaneously. The order relation between intervals in this situation is a partial order so that the solutions of (11) obtained are Pareto optimal solutions.

\section{SOLUTION APPROACH}

In order to solve the multi-objective optimization problem defined in eq.(11), we employ (1) FV scheme to calculate $\mathbb{E}\left(C\left(T_{\text {miss }}, \boldsymbol{H}, \boldsymbol{T} \mid \boldsymbol{\theta}, \boldsymbol{x}_{\boldsymbol{L}}^{*}\right)\right)$; (2) two DEs to calculate the upper and lower bounds of $\left[\mathbb{E}\left(C\left(T_{\text {miss }}, \boldsymbol{H}, \boldsymbol{T} \mid[\boldsymbol{\theta}],\left[\boldsymbol{x}_{\boldsymbol{L}}^{*}\right]\right)\right)\right]$, using the FV scheme for fitness evaluation; (3) NSDE to find the Pareto-optimal maintenance policy for $\boldsymbol{H}$ and $\boldsymbol{T}$, aiming at optimizing the interval produced by the two DEs. The meta-heuristic algorithm DE is chosen as the solution approach 
because 1) PDMP model is highly complex and non-linear and 2) $\mathrm{DE}$ is fit to optimize continuous decision variables.

\section{A. FV for solving PDMP}

To obtain $\mathbb{E}\left(C\left(t, \boldsymbol{H}, \boldsymbol{T} \mid \boldsymbol{\theta}, \boldsymbol{x}_{\mathbf{L}}^{*}\right)\right), p_{t}^{\boldsymbol{Z}_{k}}(d \boldsymbol{z}=(d \boldsymbol{x}, i) \mid \boldsymbol{\theta})$ of PDMPs need to be calculated at first. Instead of using Monte Carlo simulation, which shows high computational burden, we employ FV scheme which lead to results comparable to Monte Carlo simulation, but in significantly shorter computing times [23]. FV scheme gives an approximate solution by discretizing the state space and the time space of PDMP. An explicit FV scheme, developed by Cocozza-Thivent et al. [26], is employed in this work.

\section{B. DE approach}

DE is a simple and efficient heuristic approach for single-objective global optimization, originally developed by Store and Price [25] for continuous problems. It often shows better performance than alternative optimization algorithms, e.g. genetic algorithms. The procedure of DE is briefly presented as follows:

Step 1: Initialize randomly the population $P$ of $N_{c} \geq 4$ target individuals over the variables space.

Step 2: Generate the mutant individuals through the following mutation equation:

$v_{i, G+1}=x_{r 1, G}+F \cdot\left(x_{r 2, G}-x_{r 3, G}\right), \forall i \in\left\{1,2, \ldots, N_{c}\right\}$ (12) where $G$ is the current iteration number, $r_{1}, r_{2}, r_{3} \in$ $\left\{1,2, \ldots, N_{c}\right\}$ are random indices satisfying $r_{1} \neq r_{2} \neq r_{3} \neq i$ and $F \in[0,2]$, determined by the user, is a constant factor controlling the amplification of $\left(x_{r 2, G}-x_{r 3, G}\right)$.

Step 3: Generate each trial individual through the following crossover equation:

$$
u_{i, G+1}^{j}=\left\{\begin{array}{l}
v_{i, G+1}^{j}, \text { if }(\operatorname{rand} \leq C R) \text { or } j=\operatorname{irand}(D) \\
x_{i, G}^{j}, \text { if }(\text { rand }>C R) \text { and } j \neq \operatorname{irand}(D)
\end{array}, j=1,2, \ldots, D\right.
$$

where $u_{i, G+1}^{j}, v_{i, G+1}^{j}$ and $x_{i, G}^{j}$ are the $j$-th parameters of the vectors $u_{i, G+1}, v_{i, G+1}$ and $x_{i, G}$, respectively; rand $\in[0,1]$ is a uniform random number; $C R \in[0,1]$ is the crossover constant, determined by the user; $D$ is the dimension of the individual vector; $\operatorname{irand}(i)$ is a uniform discrete random number in the set $\{1,2, \ldots, D\}$.

Step 4: Evaluate the target and the associated trial individuals; select the best one as the target individual for the next generation.

Step 5: Go back to step 2, if the termination criterion is not met; otherwise, stop the algorithm.

The maximum iteration number $\left(N_{\max }\right)$, maximum fitness evaluation number $\left(T_{\max }\right)$ and minimum fitness error $(e p s)$ are typically employed individually or jointly as the termination criterion.

We use two DE algorithms (DE1 and DE2) using the FV scheme for the fitness function evaluation to obtain $\underline{\mathbb{E}\left(C\left(T_{\text {miss }}, \boldsymbol{H}, \boldsymbol{T} \mid[\boldsymbol{\theta}],\left[\boldsymbol{x}_{\boldsymbol{L}}^{*}\right]\right)\right)}$ and $\overline{\mathbb{E}\left(C\left(T_{\text {miss }}, \boldsymbol{H}, \boldsymbol{T} \mid[\boldsymbol{\theta}],\left[\boldsymbol{x}_{\mathbf{L}}^{*}\right]\right)\right)}$, respectively: DE1 selects the one with smallest value as the target individual for the next generation at step 4 whereas DE2 selects the one with largest value.

\section{NSDE}

For solving the multi-objective problem formulated in eq. (11), the non-dominated sorting mechanisms are included in the single objective DE, similar to the work [24] where the non-dominated sorting mechanisms are combined with a modified binary DE (MBDE). For the details about this approach, please kindly refer to [24].

\section{Integration of methods}

These methods are integrated by using (1) FV scheme for the fitness evaluation in DE and (2) DE for the fitness evaluation in NSDE; the solution methods are integrated, for the first time, for maintenance optimization. The flowchart of the entire optimization methodology that integrates the methods mentioned above is shown in Fig. 3.

\begin{tabular}{|c|c|}
\hline \multicolumn{2}{|l|}{ NSDE } \\
\hline \multicolumn{2}{|c|}{$\begin{array}{c}\text { Set initial parameters for NSDE, DE1 and DE2. } \\
\text { Initialize the initial population } P_{1} \text { of } N_{c}^{1} \geq 4 \text { target individuals for } \boldsymbol{H}, \boldsymbol{T}\end{array}$} \\
\hline $\begin{array}{c}\text { Initialize the initial population } P_{2} \text { of } \\
N_{C}^{2} \geq 4 \text { target individuals for } \boldsymbol{\theta} \text { and } \boldsymbol{x}_{L}^{*}\end{array}$ & $\begin{array}{l}\text { Initialize the initial population } P_{3} \text { of } \\
N_{C}^{2} \geq 4 \text { target individuals for } \boldsymbol{\theta} \text { and } \boldsymbol{x}_{L}^{*} \text {. }\end{array}$ \\
\hline$\rightarrow \begin{array}{c}\text { Generate the population } P_{2}^{*} \text { of } \\
\text { trial individuals of } P_{2}\end{array}$ & $\begin{array}{c}\text { Generate the population } P_{3}^{*} \text { of } \\
\text { trial individuals of } P_{3} \text {. }\end{array}$ \\
\hline $\begin{array}{c}\text { Given each individual in } P_{1} \text {, calculate } \\
\mathbb{E}\left(C\left(T_{\text {miss }}, \boldsymbol{H}, \boldsymbol{T} \mid \boldsymbol{\theta}, \boldsymbol{x}_{L}^{*}\right)\right) \text { for each } \\
\text { individual in } P_{2} \text { and } P_{2}^{*} \text { by } \mathbf{F V} \text { scheme. } \\
\text { Select the one with smaller value as } \\
\text { the individual of } P_{2} \text { for the next } \\
\text { generation. }\end{array}$ & $\begin{array}{l}\text { Given each individual in } P_{1} \text {, calculate } \\
\mathbb{E}\left(C\left(T_{\text {miss }}, \boldsymbol{H}, \boldsymbol{T} \mid \boldsymbol{\theta}, \boldsymbol{x}_{\boldsymbol{L}}^{*}\right)\right) \text { for each } \\
\text { individual in } P_{3} \text { and } P_{3}^{*} \text { by } \mathbf{F V} \text { scheme. } \\
\text { Select the one with bigger value as } \\
\text { the individual of } P_{3} \text { for the next } \\
\text { generation. }\end{array}$ \\
\hline$\underbrace{\text { Tes }}_{\text {No }}$ DEn & 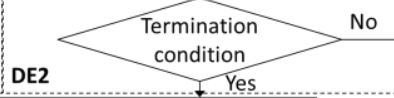 \\
\hline \multicolumn{2}{|c|}{ Obtain $\left[\mathbb{E}\left(C\left(T_{\text {miss }}, \boldsymbol{H}, \boldsymbol{T} \mid[\boldsymbol{\theta}],\left[\boldsymbol{x}_{\boldsymbol{L}}^{*}\right]\right)\right)\right]$ for each individual in $P_{1}$} \\
\hline \multicolumn{2}{|c|}{$\begin{array}{l}\text { Rank the population } P_{1} \text { by performing fast non-dominated sorting. } \\
\text { Identify the ranked non-dominated fronts. }\end{array}$} \\
\hline \multicolumn{2}{|c|}{$\begin{array}{c}\text { Apply binary tournament selection to } P_{1} \text { to } \\
\text { generate intermediate population } Q_{1} \text {. }\end{array}$} \\
\hline \multicolumn{2}{|c|}{ Generate the offspring population $P_{1}^{*}$ through crossover and mutation. } \\
\hline \multicolumn{2}{|c|}{ Obtain $\left[\mathbb{E}\left(C\left(T_{\text {miss }}, \boldsymbol{H}, \boldsymbol{T} \mid[\boldsymbol{\theta}],\left[\boldsymbol{x}_{\boldsymbol{L}}^{*}\right]\right)\right)\right]$ for each individual in $P_{1}^{*}$ by DE1 and DE2. } \\
\hline \multicolumn{2}{|c|}{$\begin{array}{c}\begin{array}{c}\text { Rank the population } R_{1}=P_{1} \cup P_{1}^{*} \text { by performing fast non-dominated sorting. } \\
\text { Identify the ranked non-dominated fronts. }\end{array} \\
\end{array}$} \\
\hline \multicolumn{2}{|c|}{ Select the $N_{c}^{1}$ best solutions from the sorted union population to update $P_{1}$. } \\
\hline
\end{tabular}

Fig. 3. Flowchart of the proposed optimization methodology.

The whole proposed framework is illustrated in Fig. 4.

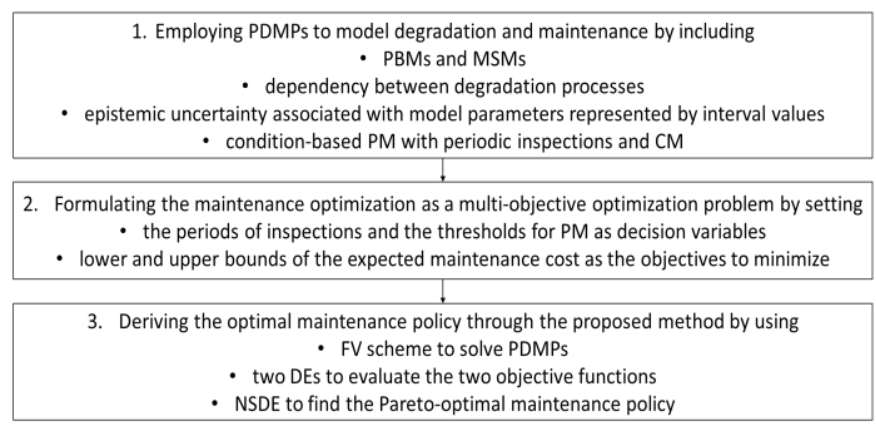

Fig. 4. The proposed framework for modeling and optimizing maintenance in systems considering epistemic uncertainty and degradation dependency. 
The proposed method is scalable to larger systems for the following reasons: "(1) FV scheme is scalable since its computational expenses and memory requirement depend almost linearly on the number of meshes partitioning the state space, which is chosen by the users. For higher dimensional problems, we can control the number of meshes to relieve the computational burden. Note that in some cases the high dimensional problem can be decomposed into several low dimensional ones mutually independent with each other. Then, the FV schemes can be run on low dimensional problems in parallel. Additionally, the computation time can be reduced via reducing the number of meshes set in FV schemes and the amount of memory required can be reduced via using sparse matrices. These issues will be investigated in future research. (2) DEs and NSDE are scalable because their simulation time lengths are mainly determined by the fitness valuation time, i.e. FV computation, and the number of generations that is independent of the system size."

\section{CASE STUDY}

In this case study, a system composed of a centrifugal pump and a pneumatic valve in series is considered, which is part of the residual heat removal system of a nuclear power plant. The system is functioning until one of the two components fails. The illustrative example is taken from the real-world residual heat removal system operated by Électricité de France (EDF) without loss of generality, which addresses the key points of interest for maintenance optimization problems: degradation dependency and epistemic uncertainty. In addition, components (degradation) states are determined by both discrete and continuous processes. The degradation dependence is considered, as a result of discussions with EDF experts: the degradation of the pump can lead it to vibrate, which will, in turn, cause the vibration of the valve and, therefore, aggravate the degradation process of the latter. This problem motivates the proposition of the maintenance optimization framework under discussion with the experts from $\mathrm{EDF}$, which tries to cope with complex situations in industrial applications. For confidentiality, the values of the model parameters and the costs of the maintenance policy presented below are altered so as to render them fictitious.

The pump has one degradation process, related to the external leakage, which is modeled by a MSM. Its degradation state at time $t$ is denoted by $Y_{p}(t)$ which takes values from $S_{p}=\left\{{ }^{\prime} 0\right.$ ' ' 1 ', ' 2 ', ' 3 ' $\}$. The pump is functioning until it reaches the state ' 0 '. Due to degradation, the pump begins to vibrate when it reaches ' 2 ' and it vibrate more intensely when it reaches ' 1 '. $\lambda_{32}, \lambda_{21}$ and $\lambda_{10}$ are the transition rates.

The scheme of the valve is shown in Fig 5.

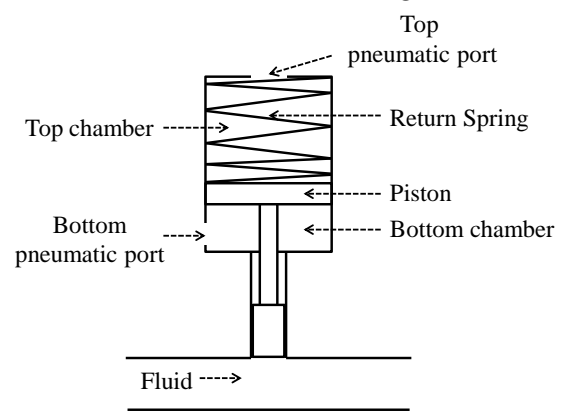

Fig. 5. Scheme of the valve [17].

The most influential degradation mechanism of the valve is the external leak at the actuator connections to the bottom pneumatic port caused by corrosion [17].

Let $D_{b}(t)$ denote the external leak size (measured by $\mathrm{m}^{2}$ ) at the actuator connections to the bottom pneumatic port caused by corrosion at time $t$, its development is characterized as follows:

$$
\dot{D_{b}}(t)=\omega_{b}\left(1+\beta_{Y_{p}(t)}\right)
$$

where $\omega_{b}$ represents the original wear coefficient at normal operating condition and $\beta_{Y_{p}(t)}$ represents the relative increment of the wear coefficient induced by the vibration of the pump. The leak can increase the opening time of the valve. The failure threshold of $D_{b}(t)$, denoted by $D_{b}^{*}$, is defined as the value that if $D_{b}(t)>D_{b}^{*}$, then, the valve cannot be fully opened within $15 \mathrm{~s}$.

The degradation of the valve $\boldsymbol{L}=\left\{L_{1}\right\}$ is described by PBM and the degradation of the pump $\boldsymbol{K}=\left\{K_{1}\right\}$ is described by MSM. The degradation processes of the whole system can be obtained:

$$
\boldsymbol{Z}(t)=\left(\begin{array}{c}
D_{b}(t) \\
Y_{p}(t)
\end{array}\right) \in \mathbb{R}^{+} \times S_{p}
$$

The failure state set of $\boldsymbol{Z}(t)$ is $\mathcal{F}=[0,+\infty) \times\left\{{ }^{\prime} 0^{\prime}\right\} \cup$ $\left[D_{b}^{*},+\infty\right) \times\left\{1^{\prime},{ }^{\prime} 2^{\prime}, 3^{\prime}\right\} \quad . \quad \boldsymbol{\theta}_{\boldsymbol{L}}=\left\{\omega_{b}\right\} \times\left\{\beta_{2}\right\} \times\left\{\beta_{1}\right\}, \boldsymbol{\theta}_{\boldsymbol{K}}=$ $\left\{\lambda_{32}\right\} \times\left\{\lambda_{21}\right\} \times\left\{\lambda_{10}\right\}$ and $x_{L}^{*}=\left\{D_{b}^{*}\right\}$ are the uncertain parameters. As an example, a relative uncertainty of $\pm 10 \%$ of the original parameters values has been considered to assign their interval values. Their interval values are shown in Table I, under accelerated aging conditions.

Table I The interval values of the uncertain parameters in PDMP

\begin{tabular}{|c|c|}
\hline Parameter & Interval value \\
\hline$\omega_{b}$ & {$\left[\omega_{b}\right]=[9 \mathrm{e}-9,1.1 \mathrm{e}-8] \mathrm{m}^{2} / \mathrm{s}$} \\
\hline$\beta_{2}$ & {$\left[\beta_{2}\right]=[9 \%, 11 \%]$} \\
\hline$\beta_{1}$ & {$\left[\beta_{1}\right]=[18 \%, 22 \%]$} \\
\hline$\lambda_{32}$ & {$\left[\lambda_{32}\right]=[2.7 \mathrm{e}-3,3.3 \mathrm{e}-3] \mathrm{s}-1$} \\
\hline$\lambda_{21}$ & {$\left[\lambda_{21}\right]=[2.7 \mathrm{e}-3,3.3 \mathrm{e}-3] \mathrm{s}-1$} \\
\hline$\lambda_{10}$ & {$\left[\lambda_{10}\right]=[2.7 \mathrm{e}-3,3.3 \mathrm{e}-3] \mathrm{s}-1$} \\
\hline$D_{b}^{*}$ & {$\left[D_{b}^{*}\right]=[9.54 \mathrm{e}-6,1.166 \mathrm{e}-5] \mathrm{m}^{2}$} \\
\hline
\end{tabular}

The system initial state is $\boldsymbol{Z}_{0}=\left(\begin{array}{l}D_{b}(0) \\ Y_{p}(0)\end{array}\right)=\left(\begin{array}{l}0 \\ 3\end{array}\right)$. The initial probability distribution of $\left(D_{b}(t), Y_{p}(t)\right)_{t \geq 0}, p_{0}(d \boldsymbol{z} \mid \boldsymbol{\theta})$, hence, equals to $\delta_{Z_{0}}(d z)$, where $\delta$ is the Dirac delta function.

The problem of maintenance optimization under uncertainty on the horizon of the mission time $T_{\text {miss }}$ can, then, be formulated as:

$$
\begin{gathered}
\text { Min } \frac{\mathbb{E}\left(C\left(T_{\text {miss }}, \boldsymbol{H}, \boldsymbol{T} \mid[\boldsymbol{\theta}],\left[\boldsymbol{x}_{\boldsymbol{L}}^{*}\right]\right)\right)}{\overline{\bar{E}\left(C\left(T_{\text {miss }}, \boldsymbol{H}, \boldsymbol{T} \mid[\boldsymbol{\theta}],\left[\boldsymbol{x}_{\boldsymbol{L}}^{*}\right]\right)\right)}} \\
\text { Subject to } \left.H_{L_{1}} \subseteq\right] 0, \overline{D_{b}^{*}}[ \\
\boldsymbol{H}_{K_{1}} \subseteq\left\{0^{\prime},{ }^{\prime}{ }^{\prime},{ }^{\prime}{ }^{\prime}{ }^{\prime}, 3^{\prime}\right\} \\
0 \leq T_{i} \leq T_{\text {miss }}, \forall i \in\left\{L_{1}, K_{1}\right\}
\end{gathered}
$$

The related costs affecting the maintenance policy are shown in Table II.

Table II The related costs of the maintenance policy 


\begin{tabular}{|c|c|}
\hline Task & Cost $(\mathrm{k} €)$ \\
\hline Inspection of pump & 2 \\
\hline PM of pump & 5 \\
\hline CM of pump & 10 \\
\hline Inspection of valve & 2 \\
\hline PM of valve & 5 \\
\hline CM of valve & 10 \\
\hline Penalty & 1000 \\
\hline
\end{tabular}

\section{RESULTS}

At first, the proposed method has been run 150 generations to obtain the Pareto optimal maintenance policies, with the following parameter values: $N_{c}^{1}$ is set to $20 ; N_{c}^{2}$ and $N_{c}^{3}$ are set to $10 ; T_{\text {miss }}$ is set to $1000 \mathrm{~s}$ following the accelerated aging condition explained in our previous work [18]; $C R$ is set to 0.7 for DE1 and DE2, and is set to 0.8 for NSDE; $F$ is set to 0.5 for DE1 and DE2, and is set to 1 for NSDE. The values of $N_{c}^{1}, N_{c}^{2}$ and $N_{c}^{3}$ are chosen with respect to the computational complexity of the FV scheme and the limited computational resources. The parameters of DE1, DE2 and NSDE are determined after trials on different values.

The obtained Pareto front is shown in Fig. 6, i.e. lower and upper bounds of the maintenance cost. We can see from the figure that ignoring epistemic uncertainty may lead to substantial underestimation of maintenance cost. For example, maintenance policy (a') could be the best option suggested by the framework without epistemic uncertainty, because it possesses the lowest maintenance cost. However, it may result to $1000 \mathrm{k} €$ penalty cost due to the system failure whose probability has been covered in the epistemic uncertainty estimate. Therefore, the proposed framework can help decision makers to choose appropriate maintenance policies which are neither too conservative nor too optimistic. Note that epistemic uncertainties due to incomplete or imprecise information is always unavoidable in industrial applications. It must be reflected and accounted for in the maintenance optimization framework.

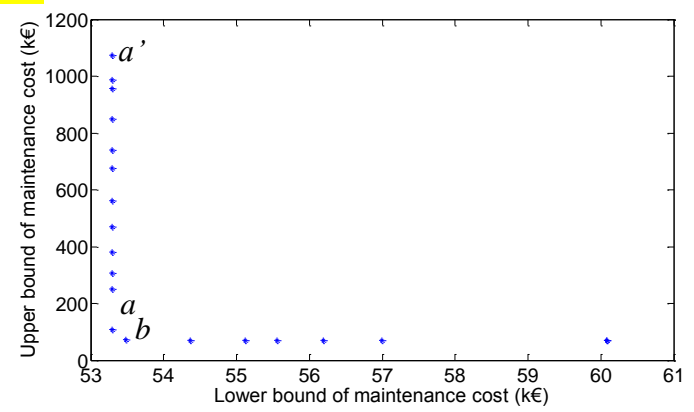

Fig. 6. The obtained Pareto front.

It is observed that the upper bounds cover a wide range whereas the lower bounds show much less variability. The solutions above $a=(53.30,108.45) \mathrm{k} €$ have big increments with respect to the upper bound, but they have nearly no difference with respect to the lower bound. The solutions to the right of $b=(53.49,72.75) \mathrm{k} €$ show nearly no difference with respect to the upper bound value. The small differences between lower bounds are due to the fact that the failures of the components rarely occurs when lower bounds are reached, so that the total cost is mainly composed of the PM costs and the inspections costs; on the contrary, the big differences between upper bounds are mainly due to the high penalty cost incurred under some situations. In practice, the solutions with very high upper bounds might not be appropriate for decision makers (DMs).

In case that the DMs intend to derive the optimal maintenance policy within a certain budget, the method proposed is also capable of dealing with this situation. For instance, we can focus on the solutions within the region $[0,100] \mathrm{k} € \times[0,100] \mathrm{k} €$. The proposed method is run with the previous configurations plus a penalty of $100 \mathrm{k} €$, this penalty is added to upper(lower) bound of a solution, whenever its lower(upper) bound exceeds $100 \mathrm{k} €$. The newly obtained Pareto front is shown in Fig. 7.

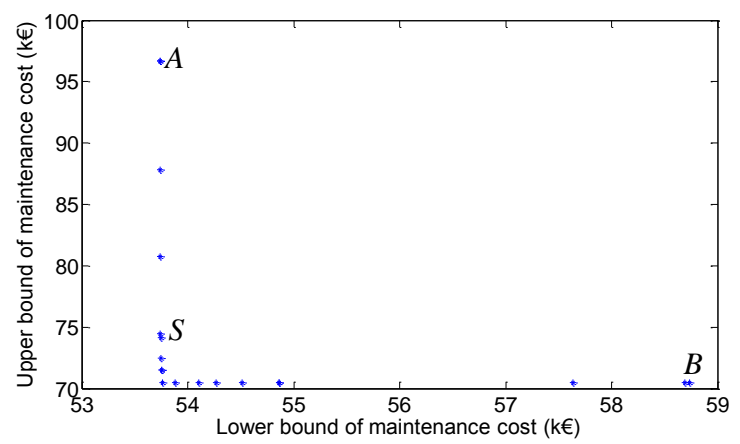

Fig. 7. The Pareto front obtained within the region $[0,100] \mathrm{k} € \times[0,100] \mathrm{k} €$.

Given the Pareto front, the DMs need eventually choose the maintenance policy according to their preferences since the solutions do not dominate each other. To simulate those common preferences of the DMs, we choose three solutions: $S$, the solution selected by the 'Min-Max' method, which selects the representative center of the Pareto front, and is among the most used ones [37]; $A$ (corresponding to a selection by decision makers who are optimistic and pay more attention to the lower bound of the cost) and $B$ (corresponding to a selection by decision makers who are conservative and pay more attention to the upper bound of the cost), which are the solutions with the minimum lower bound and minimum upper bound values, respectively. Solutions $A, B$ and $S$ can indicate three different preferences of the DMs. Detailed information on $S, A$ and $B$ is reported in Table III.

Table III Solutions $S, A$ and $B$

\begin{tabular}{|c|c|c|c|}
\hline Solution & $S$ & $A$ & $B$ \\
\hline $\begin{array}{c}\text { Lower } \\
\text { bound }\end{array}$ & $53.74 \mathrm{k} €$ & $53.73 \mathrm{k} €$ & $58.69 \mathrm{k} €$ \\
\hline $\begin{array}{c}\text { Upper } \\
\text { bound }\end{array}$ & $74.17 \mathrm{k} €$ & $96.69 \mathrm{k} €$ & $70.46 \mathrm{k} €$ \\
\hline$T_{L_{1}}$ & $773.47 \mathrm{~s}$ & $808.55 \mathrm{~s}$ & $563.00 \mathrm{~s}$ \\
\hline$T_{K_{1}}$ & $66.77 \mathrm{~s}$ & $66.77 \mathrm{~s}$ & $66.77 \mathrm{~s}$ \\
\hline$H_{L_{1}}$ & $\begin{array}{c}{\left[7.28 \mathrm{e}-6, D_{b}^{*}\right)} \\
\mathrm{m}^{2}\end{array}$ & $\begin{array}{c}{\left[7.66 \mathrm{e}-6, D_{b}^{*}\right)} \\
\mathrm{m}^{2}\end{array}$ & $\begin{array}{c}{\left[4.91 \mathrm{e}-6, D_{b}^{*}\right)} \\
\mathrm{m}^{2}\end{array}$ \\
\hline$H_{K_{1}}$ & $\left\{{ }^{\prime} 1{ }^{\prime},{ }^{\prime} 2 '\right\}$ & $\left\{{ }^{\prime} 1,{ }^{\prime} 2 '\right\}$ & $\left\{{ }^{\prime} 1{ }^{\prime}, 2 '\right\}$ \\
\hline
\end{tabular}

It can be observed that $\mathrm{S}$ and $\mathrm{A}$ have nearly the same lower bound value, whereas A has a much higher upper bound. In this case, $\mathrm{S}$ might be more appropriate than A since the small difference 0.01 could be negligible. When comparing $\mathrm{S}$ with $\mathrm{B}$, the optimistic DMs may choose B since they prefer solutions 
with smaller upper bound, whereas the conservative DMs may choose $\mathrm{S}$ if they prefer solutions with smaller lower bound.

To illustrate the convergence of the proposed method, the hypervolume indicator [38] with a reference point at $(100,100)$ and the generational distance [39] between the best Pareto fronts obtained at two consecutive generations are used. Fig. 8 (which was Fig. 9 in the original submitted manuscripte) shows their trajectories during the execution of NSDE without penalty and Fig. 9 (which was Fig. 10 in the original submitted manuscripte) shows those with penalty. There is no obvious differences between the hypervolume indicators of two figures which indicates that NSDE is able to converge in both cases after about 60 generations. The generational distance with penalty shows less variation than that without penalty, which is due to the more restricted space of decision variables with regard to the predefined budget.
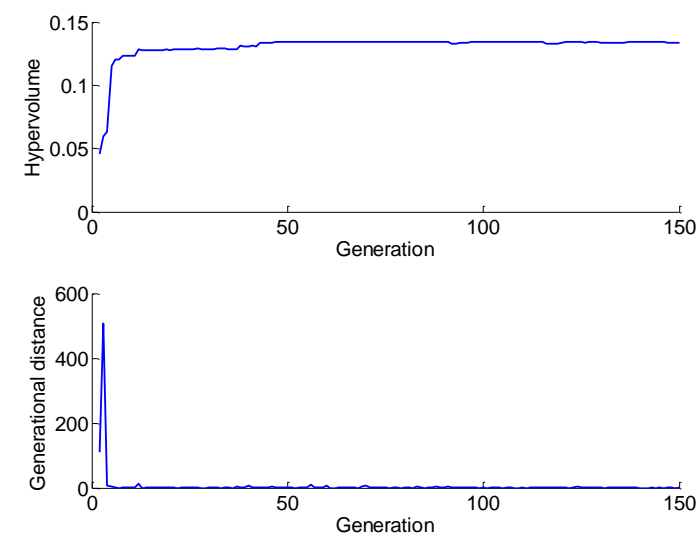

Fig. 8. The convergence plots for NSDE without penalty.

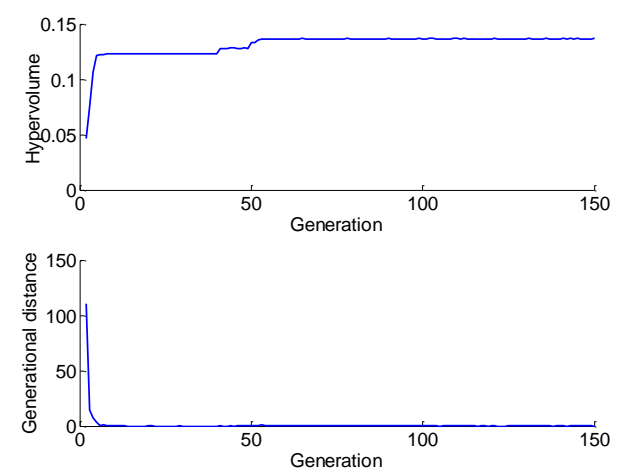

Fig. 9. The convergence plots for NSDE with penalty.

\section{CONCLUSIONS}

In this work, the problem of maintenance modeling and optimization of multi-component systems is considered, under degradation dependency and epistemic uncertainty. The component degradation processes, the condition-based PM and the CM are described through a PDMP modeling approach. Intervals are used to represent the uncertain parameters. Both the pre-scheduled periods for inspection tasks and the thresholds for PM are regarded as the decision variables in the maintenance optimization problem. Optimization is formulated in a multi-objective scheme [40] aiming at minimizing the lower and upper bounds of the interval-valued maintenance cost. To derive the optimal maintenance policy, a solution method is proposed combing FV scheme, DE approach and NSDE approach. Results on a realistic case study show the feasibility of the procedure. The main contribution of the paper is that it generalizes the existing maintenance models for multi-component systems by taking into account both degradation dependency among the components and epistemic uncertainty in the degradation models. As the future work, we plan to extend the proposed framework taking into account the economic and structural dependences between different components.

\section{REFERENCES}

[1] G. Susto, A. Schirru, S. Pampuri, S. McLoone, and A. Beghi, "Machine Learning for Predictive Maintenance: a Multiple Classifiers Approach," Industrial Informatics, IEEE Transactions on, vol. 11, pp. 812-820, 2015.

[2] L. Thomas, "A survey of maintenance and replacement models for maintainability and reliability of multi-item systems," Reliability Engineering, vol. 16, pp. 297-309, 1986.

[3] N. Rasmekomen and A. K. Parlikad, "Maintenance Optimization for Asset Systems With Dependent Performance Degradation," Reliability, IEEE Transactions on, vol. 62, pp. 362-367, 2013.

[4] S. Özekici, "Optimal periodic replacement of multicomponent reliability systems," Operations Research, vol. 36, pp. 542-552, 1988.

[5] H. Hong, W. Zhou, S. Zhang, and W. Ye, "Optimal condition-based maintenance decisions for systems with dependent stochastic degradation of components," Reliability Engineering \& System Safety, vol. 121, pp. 276-288, 2014.

[6] A. Van Horenbeek and L. Pintelon, "A dynamic predictive maintenance policy for complex multi-component systems," Reliability Engineering \& System Safety, vol. 120, pp. 39-50, 2013.

[7] W. Wang, "A Prognosis Model for Wear Prediction Based on Oil-Based Monitoring," Journal of the Operational Research Society, vol. 58, pp. 887-893, 2007.

[8] W. Wang and A. H. Christer, "Towards a General Condition Based Maintenance Model for a Stochastic Dynamic System," Journal of the Operational Research Society, vol. 51, pp. 145-155, 2000.

[9] M. Zhang, Z. Ye, and M. Xie, "A condition-based maintenance strategy for heterogeneous populations," Computers \& Industrial Engineering, vol. 77, pp. 103-114, 2014.

[10] M. Zhang, O. Gaudoin, and M. Xie, "Degradation-based maintenance decision using stochastic filtering for systems under imperfect maintenance," European Journal of Operational Research, vol. 245, pp. 531-541, 2015

[11] D. Kundu and H. Howlader, "Bayesian inference and prediction of the inverse Weibull distribution for Type-II censored data," Computational Statistics \& Data Analysis, vol. 54, pp. 1547-1558, 2010.

[12] N. Gebraeel, A. Elwany, and J. Pan, "Residual life predictions in the absence of prior degradation knowledge," Reliability, IEEE Transactions on, vol. 58, pp. 106-117, 2009.

[13] J. Lawless and M. Crowder, "Covariates and random effects in a gamma process model with application to degradation and failure," Lifetime Data Analysis, vol. 10, pp. 213-227, 2004.

[14] X. Wang, P. Jiang, B. Guo, and Z. Cheng, "Real-time Reliability Evaluation with a General Wiener Process-based Degradation Model," Quality \& Reliability Engineering, vol. 30, pp. 205-220, 2013.

[15] Z. S. Ye and N. Chen, "The Inverse Gaussian Process as a Degradation Model," Technometrics, vol. 56, pp. 302-311, 2014.

[16] M. J. Zuo, B. Liu, and D. Murthy, "Replacement-repair policy for multi-state deteriorating products under warranty," European Journal of Operational Research, vol. 123, pp. 519-530, 2000.

[17] M. Daigle and K. Goebel, "A model-based prognostics approach applied to pneumatic valves," International journal of prognostics and health management, vol. 2, pp. 1-16, 2011.

[18] Y.-H. Lin, Y. Li, and E. Zio, "Dynamic Reliability Models for Multiple Dependent Competing Degradation Processes," in ESREL 2014, pp. 775-782, 2014

[19] M. Bazu, "A combined fuzzy-logic and physics-of-failure approach to reliability prediction," Reliability, IEEE Transactions on, vol. 44, pp. 237-242, 1995 
[20] M. Laviolette and J. W. Seaman, "The efficacy of fuzzy representations of uncertainty," Fuzzy Systems, IEEE Transactions on, vol. 2, pp. 4-15, 1994.

[21] A. Khosravi and S. Nahavandi, "Load Forecasting Using Interval Type-2 Fuzzy Logic Systems: Optimal Type Reduction," Industrial Informatics, IEEE Transactions on, vol. 10, pp. 1055-1063, 2014.

[22] O. Linda and M. Manic, "Uncertainty-robust design of interval type-2 fuzzy logic controller for delta parallel robot," Industrial Informatics, IEEE Transactions on, vol. 7, pp. 661-670, 2011.

[23] Z. Chaoyue, W. Jianhui, J. P. Watson, and G. Yongpei, "Multi-Stage Robust Unit Commitment Considering Wind and Demand Response Uncertainties," Power Systems, IEEE Transactions on, vol. 28, pp. 2708-2717, 2013.

[24] Y.-F. Li, G. Sansavini, and E. Zio, "Non-dominated sorting binary differential evolution for the multi-objective optimization of cascading failures protection in complex networks," Reliability Engineering \& System Safety, vol. 111, pp. 195-205, 2013.

[25] R. Storn and K. Price, "Differential evolution-a simple and efficient heuristic for global optimization over continuous spaces," Journal of global optimization, vol. 11, pp. 341-359, 1997.

[26] C. Cocozza-Thivent, R. Eymard, and S. Mercier, "A finite-volume scheme for dynamic reliability models," IMA journal of numerical analysis, vol. 26, pp. 446-471, 2006.

[27] Y.-H. Lin, Y.-F. Li, and E. Zio, "Fuzzy Reliability Assessment of Systems with Multiple Dependent Competing Degradation Processes," Fuzzy Systems, IEEE Transactions on, vol. 23, pp. 1428-1438, 2015.

[28] A. Lorton, M. Fouladirad, and A. Grall, "A methodology for probabilistic model-based prognosis," European Journal of Operational Research, vol. 225, pp. 443-454, 2013.

[29] M. Daigle and K. Goebel, "Multiple damage progression paths in model-based prognostics," in Aerospace Conference, 2011 IEEE, pp. 1-10, 2011.

[30] D. Zwillinger, Handbook of differential equations: Gulf Professional Publishing, 1998.

[31] M. Giorgio, M. Guida, and G. Pulcini, "An age-and state-dependent Markov model for degradation processes," IIE Transactions, vol. 43, pp. 621-632, 2011.

[32] H. R. Golmakani, "Optimal age-based inspection scheme for condition-based maintenance using $\mathrm{A}^{*}$ search algorithm," International Journal of Production Research, vol. 50, pp. 7068-7080, 2012.

[33] A. Usynin, J. W. Hines, and A. Urmanov, "Uncertain failure thresholds in cumulative damage models," in Reliability and Maintainability Symposium, 2008. RAMS 2008, pp. 334-340, 2008.

[34] Y.-F. Li, E. Zio, and Y.-H. Lin, "A Multistate Physics Model of Component Degradation Based on Stochastic Petri Nets and Simulation," Reliability, IEEE Transactions on, vol. 61, pp. 921-931, 2012.

[35] S. Zhang, M. Hodkiewicz, L. Ma, and J. Mathew, "Machinery condition prognosis using multivariate analysis," in Engineering asset management, ed: Springer, pp. 847-854, 2006.

[36] P. Moussou, S. Cambier, D. Lachene, S. Longarini, L. Paulhiac, and V. Villouvier, "Vibration investigation of a French PWR power plant piping system caused by cavitating butterfly valves," ASME-PUBLICATIONS-PVP, vol. 420, pp. 99-106, 2001.

[37] A. D. Belegundu and T. R. Chandrupatla, Optimization concepts and applications in engineering: Cambridge University Press, 2011.

[38] E. Zitzler, L. Thiele, M. Laumanns, C. M. Fonseca, and V. G. Da Fonseca, "Performance assessment of multiobjective optimizers: An analysis and review," Evolutionary Computation, IEEE Transactions on, vol. 7, pp. 117-132, 2003.

[39] D. A. Van Veldhuizen and G. B. Lamont, "On measuring multiobjective evolutionary algorithm performance," in Evolutionary Computation, 2000. Proceedings of the 2000 Congress on, pp. 204-211, 2000.

[40] M. Zare, T. Niknam, R. Azizipanah-Abarghooee, and A. Ostadi, "New Stochastic Bi-Objective Optimal Cost and Chance of Operation Management Approach for Smart Microgrid," Industrial Informatics, IEEE Transactions on, vol. 12, pp. 2031-2040, 2016.

[41] Y.-H. Lin, Y.-F. Li, and E. Zio, "A Reliability Assessment Framework for Systems With Degradation Dependency by Combining Binary Decision Diagrams and Monte Carlo Simulation," IEEE Transactions on Systems, Man, and Cybernetics: Systems, vol. 46, pp. 1556-1564, 2016. 